\title{
Radiation recall dermatitis
}

\section{Claudio Guarneri MD, Biagio Guarneri MD}

A 66-year-old woman with metastatic breast cancer was admitted to hospital after presenting with progressive disease in the lungs and a painful metastatic lesion in the left ischium. Stage IV infiltrating ductal breast cancer with metastases in the lungs and bone had been diagnosed four years earlier. The disease had stabilized initially after treatment with five courses of cyclophosphamide plus doxorubicin. Two years after diagnosis, the disease was found to have progressed, and was treated with six courses of paclitaxel. The treatment with paclitaxel had concluded three months before presentation.

Radiation therapy was administered in the patient's left hip, to a total dose of 30 Grays in the anterior-posterior field. No cutaneous reaction was noted. Two weeks after conclusion of the radiotherapy, treatment with pegylated liposomal doxorubicin was started. Eighteen days after the first infusion, the patient experienced localized, painful erythema and micropapular eruption, followed by light scaling, over her left hip and gluteal areas. The lesions were confined to the field of previous irradiation (Figure 1).

Radiation recall dermatitis is an uncommon inflammatory reaction of the skin at the site of previous irradiation. The reaction is precipitated by the use of a triggering compound after radiation therapy. Although the cause and incidence are unknown, certain drugs appear to be associated with radiation recall dermatitis more frequently than others. The first description of the reaction involved its occurrence after administration of actinomycin-D. ${ }^{1}$ Since then, many other drugs - mostly chemotherapeutic agents - have been associated with the phenomenon, including 5-fluorouracil, hydroxyurea, vinblastine, methotrexate, adriamycin, etoposide, tamoxifen, bleomycin, melphalan, paclitaxel, docetaxel, gemcitabine, pegylated liposomal doxorubicin, interferon $\alpha-2 b$, antituberculous drugs and simvastatin. ${ }^{2}$

Most reports describe the lesions as maculopapular eruptions with erythema, edema, vesicle formation and desquamation. The reaction can range in severity from a mild rash to severe necrosis of the skin. Patients commonly report pruritus or pain.

By definition, such inflammatory reactions occur exclu-

From the Department of Social Territorial Medicine, Institute of Dermatology, University of Messina, Messina, Italy

CMAJ 2009. DOI:10.1503/cmaj.090320

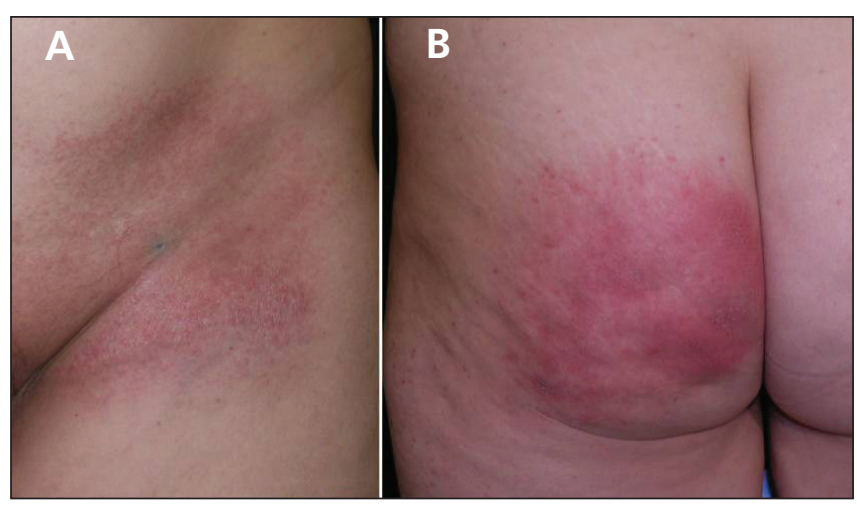

Figure 1: (A) Mild erythema with light scaling within a field of previous irradiation on the hip of a 66-year-old woman with metastatic breast cancer. (B) Erythema and edema on the patient's left buttock.

sively in fields of prior irradiation that were quiescent before the "recall." Radiation toxicity may not be evident at the time of the radiation therapy. Lesions commonly appear within days to weeks after exposure to the precipitating drug.

The dermatitis improves rapidly with use of topical steroids and, whenever possible, withdrawal of the pharmacological agent. ${ }^{3}$

This article has been peer reviewed.

Competing interests: None declared.

\section{REFERENCES}

1. D'Angio GJ, Farber S, Maddock C. Potentiation of x-ray effects by actinomycinD. Radiology 1959;73:175-7.

2. Azria D, Magné N, Zouhair A, et al. Radiation recall: a well recognized but neglected phenomenon. Cancer Treat Rev 2005;31:555-70.

3. Camidge R, Price A. Characterizing the phenomenon of radiation recall dermatitis. Radiother Oncol 2001;59:237-45.

Clinical images are chosen because they are particularly intriguing, classic or dramatic. Submissions of clear, appropriately labelled high-resolution images must be accompanied by a figure caption and the patient's written consent for publication. A brief explanation (300 words maximum) of the educational significance of the images with minimal references is required. 